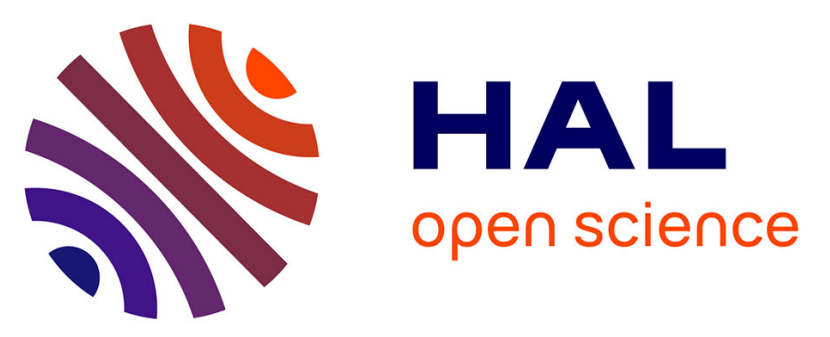

\title{
Experimental and Numerical Investigation of the Erosive Potential of a Leading Edge Cavity
}

\author{
Jean-Bastien Carrat, Regiane Fortes Patella, Jean-Pierre Franc
}

\section{To cite this version:}

Jean-Bastien Carrat, Regiane Fortes Patella, Jean-Pierre Franc. Experimental and Numerical Investigation of the Erosive Potential of a Leading Edge Cavity. International Journal of Fluid Machinery and Systems, 2019, 12 (2), pp.136-146. 10.5293/IJFMS.2019.12.2.136 . hal-02508784

\section{HAL Id: hal-02508784 \\ https://hal.science/hal-02508784}

Submitted on 19 Mar 2020

HAL is a multi-disciplinary open access archive for the deposit and dissemination of scientific research documents, whether they are published or not. The documents may come from teaching and research institutions in France or abroad, or from public or private research centers.
L'archive ouverte pluridisciplinaire HAL, est destinée au dépôt et à la diffusion de documents scientifiques de niveau recherche, publiés ou non, émanant des établissements d'enseignement et de recherche français ou étrangers, des laboratoires publics ou privés. 


\title{
Experimental and Numerical Investigation of the Erosive Potential of a Leading Edge Cavity
}

\author{
Jean-Bastien Carrat, Regiane Fortes-Patella, Jean-Pierre Franc \\ Univ. Grenoble Alpes, CNRS, Grenoble INP, LEGI, 38000 Grenoble, France \\ jb.carrat@gmail.com, regiane.fortes@legi.grenoble-inp.fr, jean-pierre.franc@legi.cnrs.fr.
}

\begin{abstract}
This paper presents a joint experimental and numerical analysis of the erosive potential of an unsteady cavity that develops at the leading edge of a two-dimensional hydrofoil and periodically sheds vapour clouds. From an experimental viewpoint, the erosive potential was characterized by pressure pulse height spectra. The hydrofoil was equipped with eight pressure sensors made of PVDF piezoelectric film that allowed the measurement of flow aggressiveness at different locations along the hydrofoil chord. It was shown that the mean peak rate over a large number of cavity pulsations exhibits a maximum at a distance from the leading edge close to the maximum cavity length. Moreover, the increase in flow aggressiveness caused by an increase in flow velocity can be explained by an increase in both amplitude and frequency of impact loads. From a numerical viewpoint, the unsteady Reynolds averaged Navier-Stokes (RANS) equations were solved using a modified k- $\varepsilon$ RNG turbulence model together with a homogeneous cavitation model within a two-dimensional approach. Flow aggressiveness was estimated from the Lagrangian derivative of the computed void fraction that allows identifying the regions of collapse of vapour structures. Three different critical regions from an erosive viewpoint were numerically identified. Apart from the region of collapse of the shed cloud (which was not instrumented in the present study), the computations showed a maximum of aggressiveness around the maximum cavity length as found experimentally. Another region of high aggressiveness closer to the leading edge and associated to the upward movement of the re-entrant jet was predicted by the present numerical model but not confirmed experimentally, which probably shows the limitation of a two-dimensional approach.
\end{abstract}

Keywords: Fluids dynamics, Cavitation erosion, Numerical calculations, Experimental measurements, Unsteady pressure measurements, PVDF sensors.

\section{Introduction}

The understanding of the cavitation erosion phenomenon and the prediction of material damage are a major challenge for hydraulic engineers and scientific researchers. On the fluid side, a key issue is to be able to estimate the erosive potential (also called hydrodynamic aggressiveness or cavitation intensity) of a cavitating flow. Numerous efforts have been made over the years in order to develop tools for quantifying the aggressiveness of a cavitating flow and define this concept more clearly.

One of the first techniques was proposed by Knapp [1] and consisted in pitting tests. Pitting tests are short duration tests that reveal the cavitation bubbles collapsing close to the wall from the plastic deformations they generate on a relatively soft ductile material. Knapp proposed to use the pitting rate as an indicator of the flow aggressiveness.

Later, the development of surface measuring techniques made it possible to improve the analysis of pitting tests by determining the precise shape of the pits [2,3]. More advanced definitions of the hydrodynamic aggressiveness have then been proposed such as distributions in pit diameter that separate the contribution to cavitation erosion of the smallest pits that are very numerous from that of the largest pits that are generated by extreme and rare events.

More recently, inverse techniques based on solid mechanics computations using the Finite Element Method (FEM) have been developed in order to assess the hydrodynamic loading conditions (typically in GPa) that would generate each pit identified in a pitting test $[4,5]$. Flow aggressiveness can then be characterized by the distribution in amplitude and size of the hydrodynamic impact loads due to bubble collapses. One of the advantages of this technique is that such a distribution can be applied numerically on the material surface in a repetitive way in order to simulate its response to a long duration exposure to the cavitation field, which will offer the possibility of computing mass loss as a function of exposure time provided a suitable damage model is introduced in the simulation. This way of measuring the flow aggressiveness uses the material itself as a kind of pressure sensor.

* Part of this paper was presented at the 29th IAHR Symposium on Hydraulic Machinery and Systems, held at Kyoto, Sept. 16-21st, 2018. 
Other techniques based on the use of conventional pressure sensors were also developed to characterize the flow aggressiveness [6]. Because of the generally much larger size of the pressure sensor compared to the impacted area, the impact load cannot be measured in pressure unit but in force unit. Flow aggressiveness is then characterized by the distribution in amplitude of impact loads also known as pressure pulse height spectrum as introduced initially by Hammitt $[7,8]$. Such an experimental technique is used in the present paper.

The same concept of impact load spectra has also been used in some numerical approaches such as [9] that succeeded to compute it and to obtain a good quantitative agreement between predicted and measured impact load spectra.

Several experimenters such as [10,11] introduced the energy of the individual cavitation impacts that is supposed to be proportional to the square of the impact load and introduced an accumulated impact energy by computing the summation of the energies of all identified impacts. The interest of this simple indicator of the flow aggressiveness is that the volume loss correlates linearly with this accumulated impact energy.

The numerical computation of impact load spectra [9] requires to resolve individual vapour structures and the shock waves induced by their collapse, which is often challenging in terms of computational resources. Simpler approaches such as [12] were also developed that do not seek to capture the dynamics of the smaller vapour structures responsible for cavitation erosion but are based on the computation of the void fraction field. This is the technique used in the present paper which is more widely discussed in Section 5.

The cavitating flow on the hydrofoil studied in this paper was thoroughly investigated from a joint numerical and experimental analysis. A detailed description of the cavity dynamics was carried out and results concerning the cavity length evolution, the shedding frequencies and corresponding Strouhal numbers were presented in [12]. In this paper, the focus is given to experimental and numerical evaluations of cavitation aggressiveness.

The hydrofoil geometry is described in section 2, as well as the experimental set-up. The most original aspects concern unsteady pressure measurements based on a line of 8 piezoelectric PVDF sensors (Polyvinylidene Fluoride). Experimental results are given in section 3. Unsteady cavitating flow simulations have been carried out using an in-house two-dimensional (2D) code presented in section 4. From CFD results, the flow aggressiveness has been evaluated by applying the prediction model proposed by Fortes-Patella et al. [13] and synthetized in section 5. Local analyses of unsteady and mean cavitation intensity are also presented and some comparisons with experimental measurements are given in section 6 .

\section{Hydrofoil geometry and experimental set-up}

Figure 1(a) shows the hydrofoil used in this study. It is symmetrical with a chord length $\mathrm{c}=100 \mathrm{~mm}$ and a maximum relative thickness of $12 \%$. Its rotation axis is at mid chord. The hydrofoil has been designed with a large flat area located between $27 \%$ and $70 \%$ of the chord length, which provides room to set up a matrix of PVDF pressure sensors. A line of eight pressure sensors aligned in the flow direction was mounted at midspan in order to make pressure pulse measurements at different locations around cavity closure that is expected to be the most aggressive region of the cavitating flow (Fig. 1(b)).

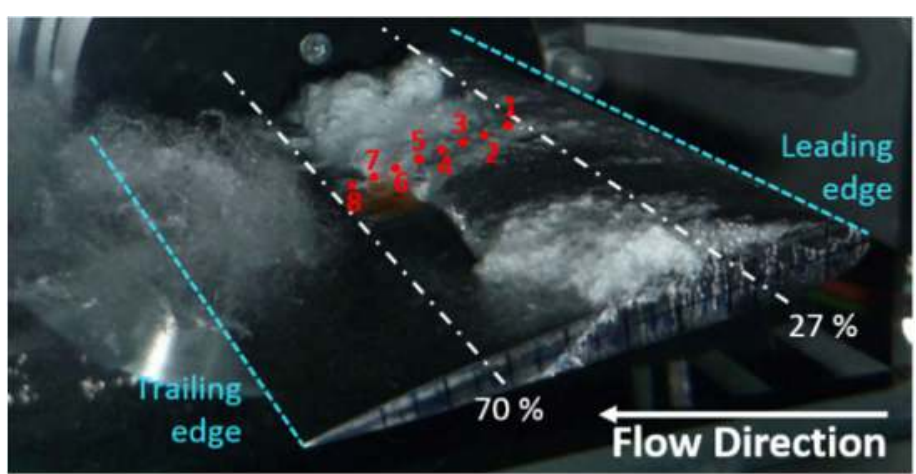

(a)

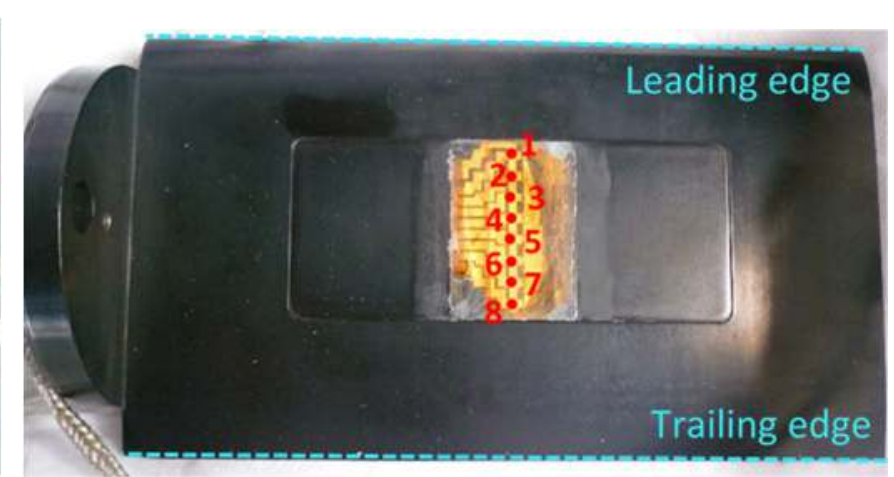

(b)

Fig. 1 Instrumented hydrofoil (a) under cavitating conditions; (b) top view.

Table 1 Sensor center positions

\begin{tabular}{c|c|c|c|c|c|c|c|c}
\hline \hline Sensor & 1 & 2 & 3 & 4 & 5 & 6 & 7 & 8 \\
\hline Position $[\mathrm{x} / \mathrm{c}]$ & 0.31 & 0.36 & 0.41 & 0.46 & 0.51 & 0.56 & 0.61 & 0.66 \\
\hline
\end{tabular}

Experiments have been conducted in the water tunnel of the LEGI laboratory (Fig. 2). Cavitation number $\sigma$ is evaluated by:

$$
\sigma=\frac{p_{u p}-p_{v}}{1 / 2 \rho V^{2}}
$$

where $\mathrm{p}_{\mathrm{up}}$ is the upstream pressure, $p_{v}$ the vapour pressure, $\rho$ the density and $V$ the test section inlet flow velocity. The uncertainties on $V$ and $\sigma$ are respectively of $0.01 \mathrm{~m} / \mathrm{s}$ and 0.02 .

The unsteady pressure sensors were made from piezoelectric films of thickness $e=40 \mu \mathrm{m}$. Assuming that both faces are free, the natural frequency of the first mode is $f_{N}=c_{f} / 2 e$ where $c_{f}$ is the speed of sound in the film. The lower face, although glued on the metallic hydrofoil, can be considered as free because the insulation and adhesive layers are relatively thick and have an acoustic impedance very similar to PVDF [14]. Since the speed of sound in the film is $c_{f} \cong 2200 \mathrm{~m} / \mathrm{s}$, the natural frequency is $f_{N}=27.5 \mathrm{MHz}$. Such a high natural frequency ensures a rapid response and makes PVDF pressure sensors particularly suitable for the measurement of short duration pressure pulses due to bubble collapses. 

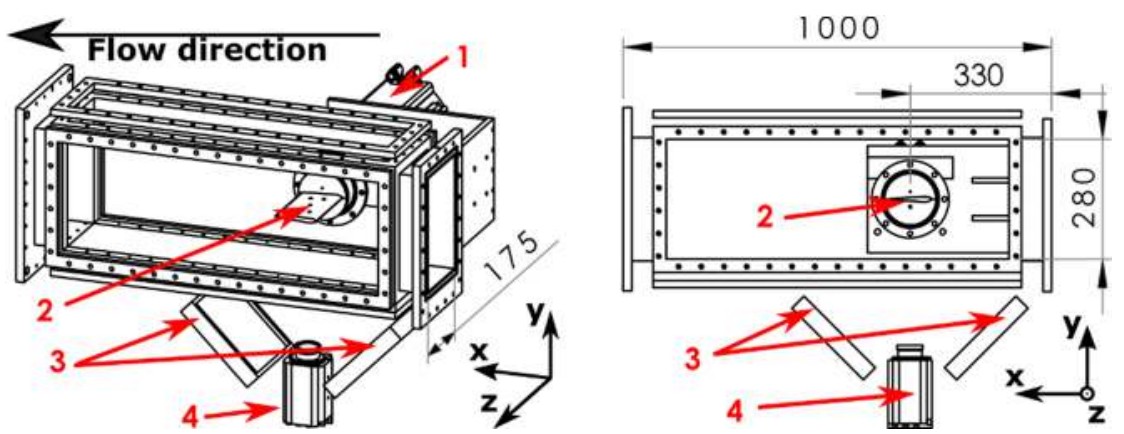

Fig. 2 Experimental set-up (1) brushless Kollmorgen motor (AKM74L); (2) hydrofoil; (3) light projectors; (4) high speed camera (MIRO C310). The side of the test section is in Plexiglas. High speed videos have been taken from the bottom at a framerate of $3200 \mathrm{fps}$.

The line of eight square pressure sensors, each of size $2 \mathrm{~mm}$ x $2 \mathrm{~mm}$, was fabricated from a metallized PVDF film by the conventional technique of chemical etching. The upper and lower surfaces were protected and electrically insulated by means of a Kapton layer of 60 $\mu \mathrm{m}$ in thickness. When bonded on the hydrofoil, the final sensors had a total thickness of about $200 \mu \mathrm{m}$. The distance between the centers of two consecutive pressure sensors is $5 \mathrm{~mm}$ (Table 1).

Each sensor in final form (including connecting cables) was dynamically calibrated by the ball-dropping method [15]. Pressure sensors were calibrated in load $(\mathrm{N})$ and not in pressure $(\mathrm{MPa})$ since their sensitive surface is generally much larger than the size of impact loads due to cavitation bubble collapses so that loading is far from being uniform over the whole sensitive surface. The measured sensitivity slightly changes from one transducer to another and is on the order of $20 \mathrm{mV} / \mathrm{N}$. Pressure sensors were calibrated before and after measurements in the cavitation tunnel in order to detect any possible drift in sensitivity due to sensor ageing or damage by cavitation. The maximum observed decrease in sensitivity is about $20 \%$. It affects primarily the most heavily loaded sensors. The ball-dropping method also allows estimating the mutual interference between neighboring sensors, which was smaller than $5 \%$ in terms of the ratio of signal amplitudes.

This paper presents results for an angle of attack of 4 deg., flow velocities between 8 and $10.4 \mathrm{~m} / \mathrm{s}$ and a cavitation number around 1.

\section{Experimental results and analyses}

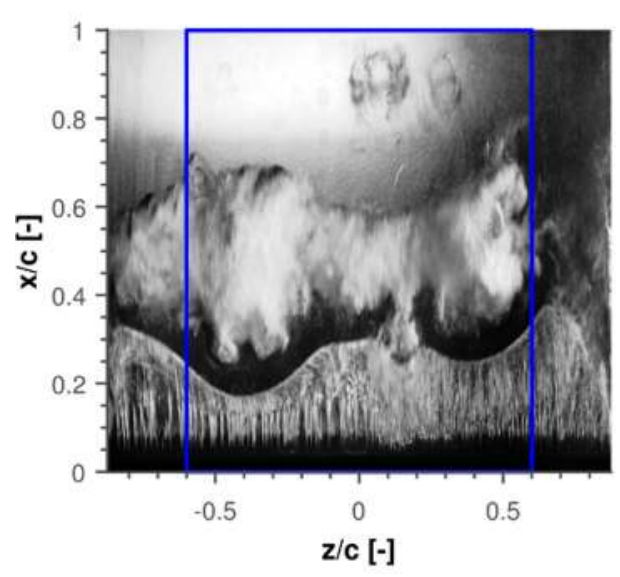

(a)

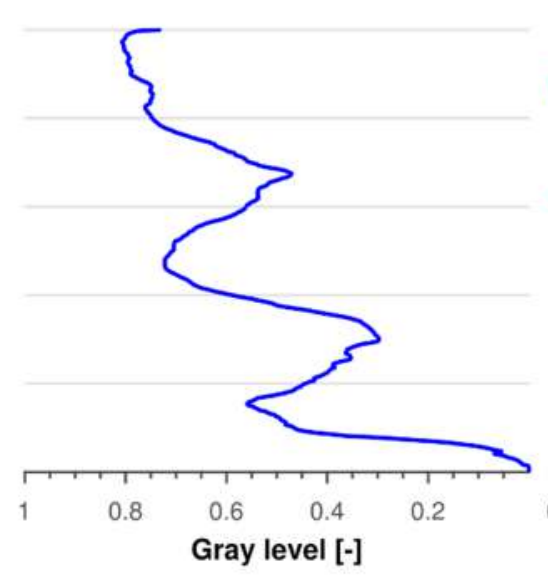

(b)

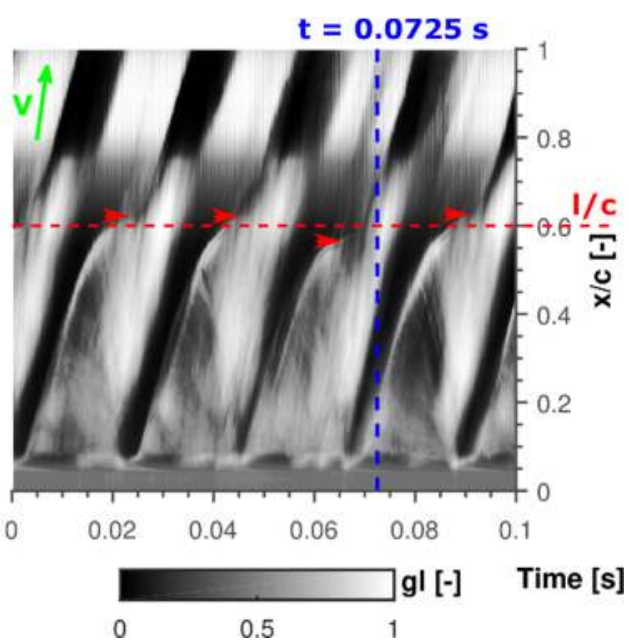

(c)

Fig. 3 (a) Snapshot at $t=0.0725 \mathrm{~s}$ with a blue rectangle representing the reduced snapshot. (b) Mean gray profile in the reduced snapshot at $t=0.0725 \mathrm{~s}$. (c) Space-time diagram of gray level; black and white are respectively a gray level of 0 and 1 .

(Angle of attack: $4 \mathrm{deg}$., $V=8 \mathrm{~m} / \mathrm{s}$ and $\sigma=1.1$ ).

Figure 3 illustrates the experimental post-treatment based on high speed videos. Each video corresponds to 8300 frames and $2.5 \mathrm{~s}$ duration and includes a large number of shedding cycles (around one hundred). To avoid any side effects due to the tunnel walls, the cavitating flow is studied only in a reduced window $120 \mathrm{~mm}$ wide, centered on the hydrofoil (the blue rectangle in Fig. 3(a)). The mean gray profile along the chord is then estimated by the spatial averaging of the gray profile over the hydrofoil span ( $\mathrm{z}$ direction) in the reduced window as shown in Fig. 3(b) for $t=0.0725 \mathrm{~s}$. It is calculated for every frame and provides the space-time diagram of the mean gray level shown in Fig 3(c). This kind of analyses allows estimating the maximum cavity length and the shedding frequency for different cavitating conditions [12]. In Fig 3(c), the maximum cavity length is indicated by red arrows. It corresponds to the moment when the overall cavity is detached from the hydrofoil surface and appears to be slightly changing between shedding cycles. The maximum cavity length defined in this paper is an average and is $l / c=0.60$ with an uncertainty of 0.05 in the case of Fig.3.

Impact load measurements were done with a sampling frequency of $10 \mathrm{MHz}$. An example of unsteady pressure signal obtained by PVDF sensors exposed to a cavitating flow is given in Fig. 4. The signal is post-processed by using the MATLAB ${ }^{\text {TM }}$ function 
"findpeaks". The function gives the amplitude, position and duration at mid height of every peak above a given threshold of 1 N, chosen just above the overall noise.

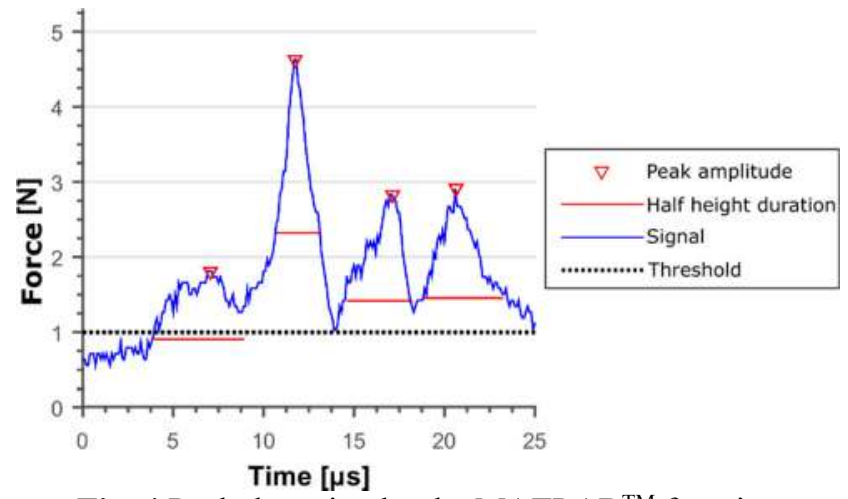

Fig. 4 Peak detection by the MATLAB ${ }^{\mathrm{TM}}$ function "findpeaks" on the sensor $7(x / c=0.61)$.

$(\sigma=1.1, V=8 \mathrm{~m} / \mathrm{s}$, angle of attack: $4 \mathrm{deg}$. and $l / c=0.55 \pm 0.05)$

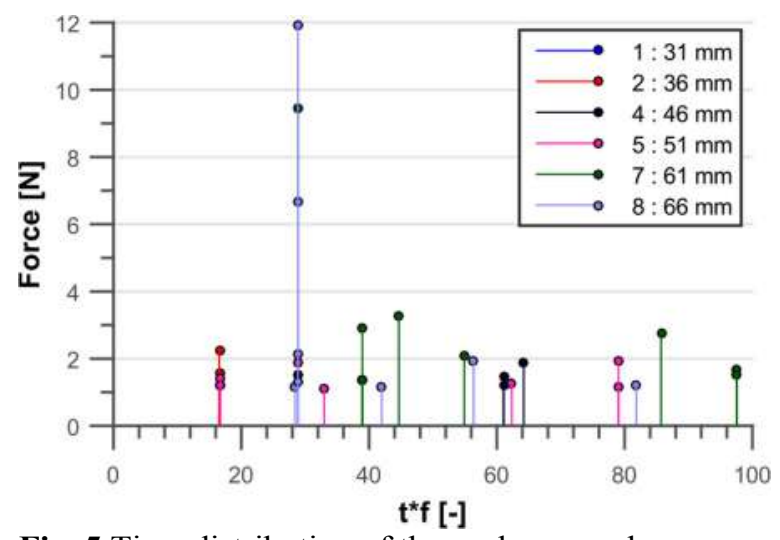

Fig. 5 Time distribution of the peaks on each pressure transducer. In horizontal axis, the time scale $t$ is multiplied by the shedding frequency f so that the horizontal axis represents the number of shedding cycles.

$(\sigma=1.1, V=8 \mathrm{~m} / \mathrm{s}$, angle of attack: $4 \mathrm{deg}$. and $l / c=0.55 \pm 0.05)$

From these measurements, one can analyze the time distribution of the peaks on every pressure sensor, as indicated in Fig. 5. Most of the peaks are observed on sensors 5,7 and 8 located around the cavity closure, estimated at $l / c=0.55 \pm 0.05$ for this operating point. During 100 sheddings, there are almost no peaks measured by sensors 1 and 2 which are within the leading edge cavity. Although the operating point does not change over time, the peak amplitude on a given sensor is not the same for all shedding cycles, which tends to prove that the smaller scale vapour structures responsible for impact loads are not only controlled by the larger scale structures. According to Fig. 5, there is also far less than one pulse per shedding cycle for the relatively high threshold of $1 \mathrm{~N}$ chosen in this work. Similar results were observed from high-speed videos and pitting tests on thin metal foils in a Venturi [16] and for a cavitating jet [17]. Both studies report that there was not a pit for every shedding cycle.

The total peak rate $\dot{N}_{t}$ for all peaks above the threshold has been plotted in Fig. 6 as a function of the distance to the leading edge for three different velocities and a constant cavitation number and angle of attack. Figure 6 shows that the maximum aggressiveness is observed in the closure region of the cavity, as reported by numerous researchers [18-22]. In the present case, the erosive potential is mostly due to small scale vapour structures that are shed by the leading edge cavity. The large scale cloud that is regularly shed by the cavity generally collapses further downstream of the instrumented region and then does not contribute to the impact loads measured here (see section 5.2).

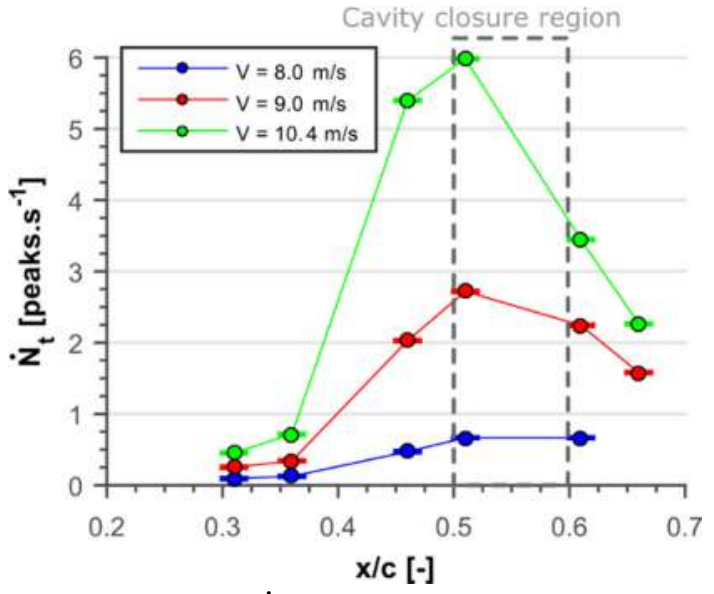

Fig. 6 Total peak rate $\dot{N}_{t}$ for a threshold of $\mathrm{F} \geq 1 \mathrm{~N}$ along the hydrofoil chord for three flow velocities $V$. The horizontal line on each point corresponds to the limits of the sensor. $(\sigma \approx 1.1$, angle of attack: 4 deg.).

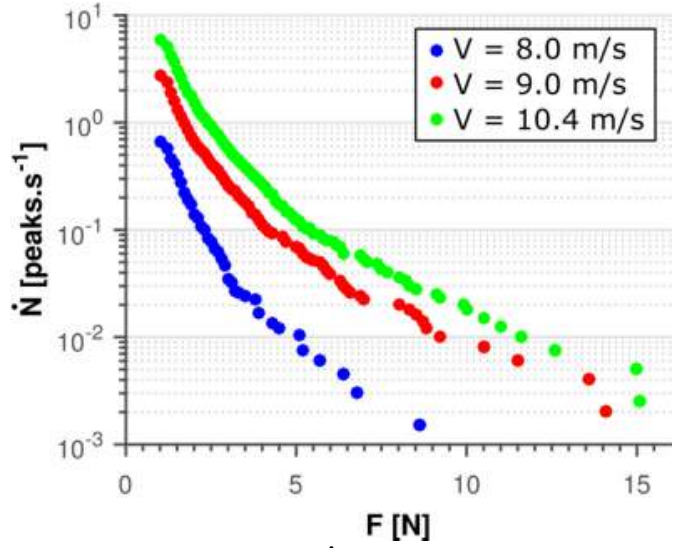

Fig. 7 Cumulative peak rate $\dot{N}$ as a function of the force $F$ on sensor $\mathrm{n}^{\circ} 5(x / c=0.51)$ for three flow velocities $V$ $(\sigma \approx 1.1$, angle of attack: $4 \mathrm{deg}$., $l / c=0.55 \pm 0.05)$.

In order to have more information on the aggressiveness of the cavitating flow, several researchers (see for instance Kim et al. [6]) proposed to plot cumulative histograms of peak rate $\dot{N}$ versus amplitude $F$. Figure 7 shows the cumulative peak rate distribution measured for three different flow velocities by pressure sensor ${ }^{\circ} 5$, located around the cavity closure where the total peak rate $\dot{N}_{t}$ is maximum (see Fig. 6). Figure 7 shows that the peak rate $\dot{N}$ significantly increases with flow velocity and that the maximum measured amplitude $F$ also increases with flow velocity. These results suggest that the increase in flow aggressiveness is due to an increase in both amplitude and frequency of impact loads, which might explain the strongly non-linear behavior of cavitation erosion damage with flow velocity. 


\section{CFD simulations}

Cavitating unsteady flows have been simulated with the in-house 2D code referred to as "IZ". The code is widely described in [23] and was applied and validated in previous studies (for example [13,24,25]) for different geometries of hydrofoils, Venturis and cascades of hydrofoils. The code solves the Unsteady Reynolds Averaged Navier-Stokes equations for a homogeneous fluid characterized by the mixture density $\rho$ defined as a function of the void ratio $\alpha$ :

$$
\rho=\alpha \rho_{v}+(1-\alpha) \rho_{l}
$$

where $\rho_{v}$ and $\rho_{l}$ are the vapour and liquid densities, respectively. Pure phases are considered incompressible.

Calculations do not consider the thermodynamic effect that is negligible in cold water and then the energy equation is not solved. The two phases are supposed to be in dynamic equilibrium in each cell so that no drift velocity is considered between liquid and vapour. The modified k- $\varepsilon$ RNG turbulence model detailed in [24] is used together with standard wall functions.

To model the cavitation phenomenon and to close the governing equation system, a barotropic state law $\rho(p)$ is used [9] that explicitly links the mixture fluid density to the local static pressure. This law is mainly controlled by its maximum slope, which is related to the minimum speed of sound $c_{\min }$ in the mixture. In the present study, we have chosen $c_{\min }=1 \mathrm{~m} / \mathrm{s}$, which proved to give good numerical predictions for cold water [10].
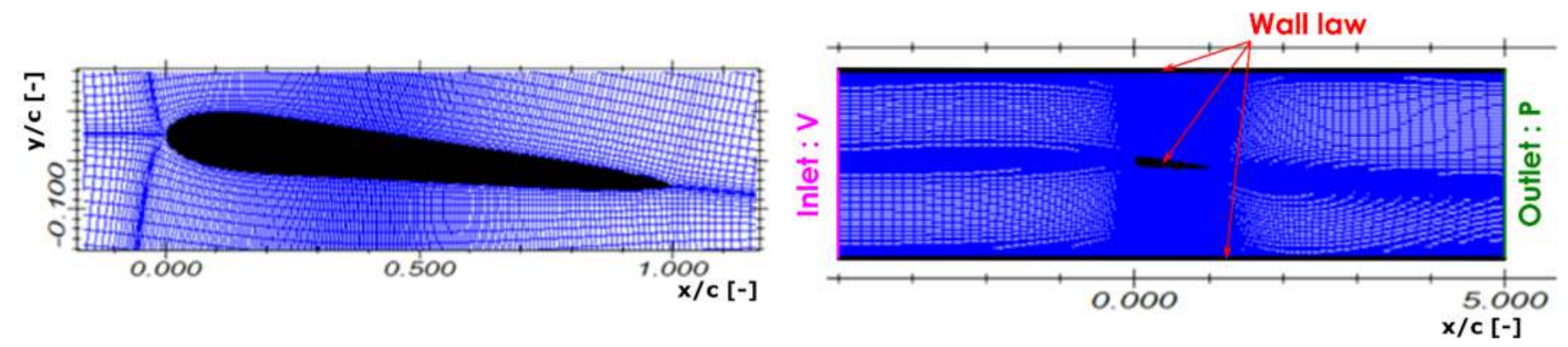

Fig. 8 Computational domain: applied mesh (320 x 159 nodes) and boundary conditions.

The computational H-grid used in the present study is illustrated in Fig. 8. The dimensionless wall distance $\mathrm{y}^{+}$of the boundary layer varies between about 20 and 50 under non-cavitating conditions, which ensures that the standard wall function can be used. Usual incompressible boundary conditions are applied: flow velocity is imposed at inlet, and the pressure (consequently, the downstream cavitation number) is fixed at outlet. The upstream cavitation number fluctuates during unsteady calculations and a numerical mean value of $\sigma$ is estimated from simulations to carry out comparisons with experiments.

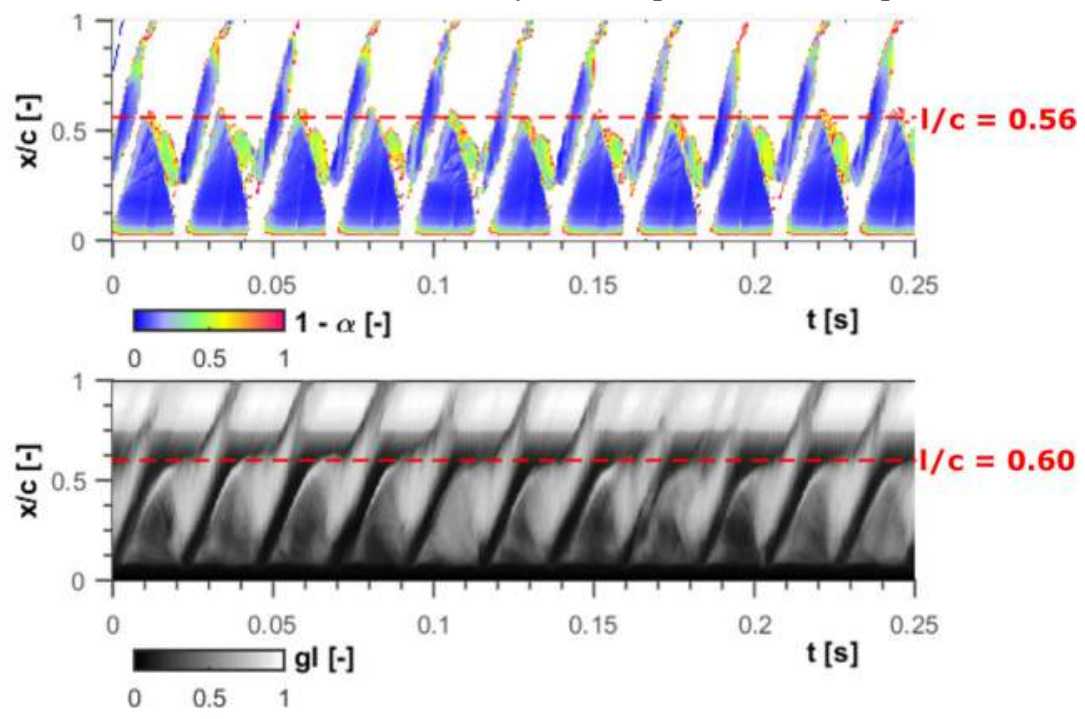

Fig. 9 Comparisons between numerical (a) and experimental (b) space-time diagrams. $l / c$ corresponds to the maximum cavity length observed. In (a), blue and white are respectively for pure vapour and liquid. The void fraction $\alpha$ shown at each point (x, $t$ ) is the minimum value of the void fraction along the line $x=$ constant perpendicular to the foil in the $(x, y)$ domain. In (b), $g l$ corresponds to gray level. Numerical shedding frequency $f=43 \mathrm{~Hz}$; experimental shedding frequency $f=48 \mathrm{~Hz}$. $(\sigma=1.1, V=8 \mathrm{~m} / \mathrm{s}$ and angle of attack: $4 \mathrm{deg}$.).

Several calculations were performed in previous work [12] considering different flow conditions. Detailed physical analyses concerning cavity dynamics were presented and the cavitating flow simulations were validated by comparisons with experimental data obtained mainly from high speed videos.

Figure 9 presents some results corresponding to the operating point ( $\sigma=1.1, V=8 \mathrm{~m} / \mathrm{s}$ and angle of attack: 4 deg.). The behavior of the partial cavity is globally well predicted by the simulations. Shedding frequencies and cavity extensions obtained experimentally and numerically are close.

In the present paper, the unsteady CFD results have been used to evaluate the cavitation aggressiveness by applying the prediction 
model proposed by [13] and described in the next section.

\section{Flow aggressiveness}

\subsection{Prediction model}

The prediction model used here is based on the energy balance approach presented in [13] and was described, tested and qualitatively validated in $[13,25,26]$ for two other hydrofoils. Based on the idea of Vogel et al. [27], the potential energy of a vapour structure is defined as the vapour volume $V_{\text {vap }}$, multiplied by a pressure imbalance $\Delta p$ :

$$
E=\Delta p V_{v a p}
$$

where $\Delta p=p-p_{v}$ is the pressure difference between the surrounding pressure $p$ and the vapour pressure $p_{v}$. From this assumption, a volumetric potential power density can be deduced by computing the Lagrangian derivative of the potential energy to take into account the fact that bubbles are moving with the fluid. The instantaneous volumetric potential power density $P$ is given by:

$$
P=\Delta p \frac{D \mathrm{E}}{D t}=\Delta p \frac{D V_{v a p}}{D t}+\mathrm{V}_{\mathrm{vap}} \frac{D \Delta p}{D t}
$$

Leclercq et al. [26] showed that the $\Delta p$ variation is negligible compared to the variation of $V_{v a p}$. In addition, only the collapse phases (i.e. $\mathrm{D} V_{\text {vap }} / \mathrm{D} t<0$ ) are taken into account in the aggressiveness evaluation since no erosion is expected during the growth phase. So, Equation (2) can be simplified into:

$$
P=-\Delta p \frac{D V_{v a p}}{D t}
$$

The minus sign is introduced in order to have a positive value.

At every time step the simulation gives the pressure $p$, velocity $V$, void fraction $\alpha$ and vapour volume $V_{v a p}$ in a cell. Since the vapour volume $V_{v a p}$ is related to the void fraction by $\alpha=V_{v a p} / V_{c e l l}$ and by using the mass conservation equation, a volumetric potential power density $P_{3 d}$ is defined in a cell by:

$$
P_{3 d}=\frac{P}{V_{\text {cell }}}=-\Delta p \frac{D \alpha}{D t}
$$

By using the mass conservation equation, eq. (4) can be written in the following form:

$$
P_{3 d}=-\Delta p \frac{\rho}{\rho_{l}-\rho_{v}} \operatorname{div}(\vec{U})
$$

To characterize the instantaneous aggressiveness along the hydrofoil chord, a potential power density per unit surface area of the wall $P_{2 d}$ is evaluated by integrating eq. (5) along a direction perpendicular to the wall:

$$
P_{2 d}=\int_{0}^{h} P_{3 d} \cdot d y
$$

where $\mathrm{h}$ is the distance to the solid wall below which the structures are estimated close enough to the wall to be aggressive. In the present work, the value of the distance $h$ has been considered large enough to take into account all the vapour structures calculated in the computational domain. The influence of this parameter in the aggressiveness prediction was evaluated in [25,26].

The temporal mean aggressiveness intensity $P_{\text {mean }}$ has been calculated for each hydrofoil surface element by considering several vapour shedding cycles ( $T_{a}$ is the analyses duration) and is defined by:

$$
P_{\text {mean }}=\frac{1}{T_{a}} \int_{0}^{T_{a}} P_{2 d} \cdot d t
$$

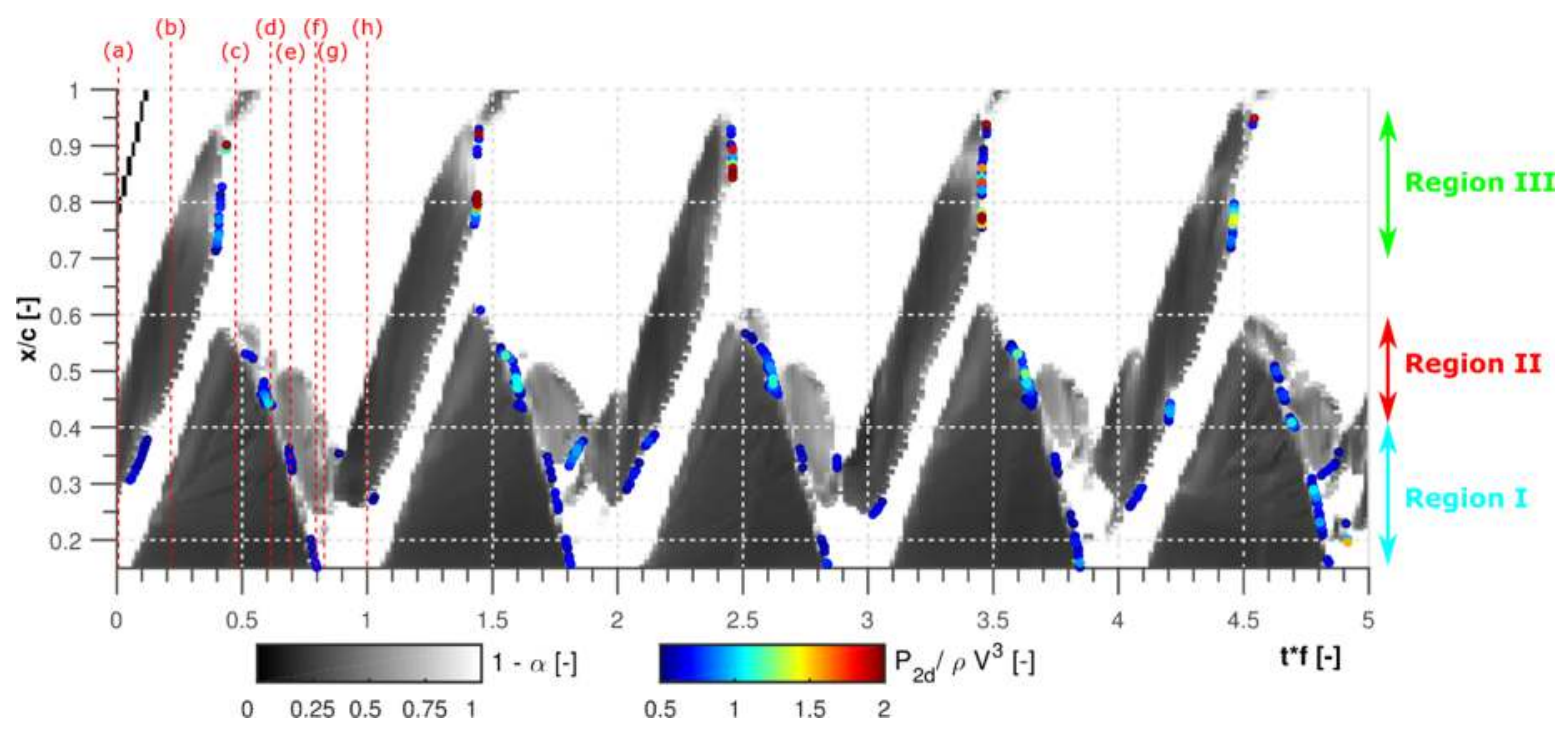

Fig. 10 Space-time diagram of the non-dimensional instantaneous aggressiveness $P_{2 d} / \rho^{*} V^{3}$ (in color) and in this case the void ratio $\alpha$ is plotted in black and white (white is liquid, black is vapour) in order to see more clearly the non-dimensional instantaneous aggressiveness $P_{2 d} / \rho^{*} V^{3}$ for five shedding cycles. The (a), (b), (c), (d), (e), (f), (g) and (h) correspond to the moments in Fig. 11.

$(\sigma=1.1, V=8 \mathrm{~m} / \mathrm{s}$ and angle of attack: $4 \mathrm{deg}$.$) .$

It was evaluated on numerical sensors located at the same positions and with the same size $2 \mathrm{~mm}$ as the experimental ones (see table 1). Numerical results are compared to experimental ones in section 6. 


\subsection{Analyses of instantaneous aggressiveness}

From numerical calculations, the non-dimensional instantaneous aggressiveness defined here by $P_{2 d} / \rho V^{3}$ can be evaluated along the chord for every time step and associated to the cavity behavior, as illustrated in Fig. 10 and 11. These local analyses allow us to identify the potentially erosive cavitation patterns and to predict the most heavily loaded regions of the hydrofoil for the considered operating point ( $\sigma=1.1, V=8 \mathrm{~m} / \mathrm{s}$ and angle of attack: 4 deg.).

In Fig. 10, only the instantaneous aggressiveness bigger than $0.5^{*} \rho^{*} V^{3}$ is plotted in color on the same graph as the space time diagram of the void ratio in black and white, white is liquid and black is vapour. As expected the instantaneous aggressiveness is directly linked to the oscillation of the cavity. From $t^{*} f=0.01$ to $t^{*} f=0.47$ in Fig. 10 or 11 , the cavity is growing, there is no instantaneous aggressiveness of more than $0.5^{*} \rho^{*} V^{3}$ due to the attached cavity. The upstream part of the cloud shed during the previous cycle is responsible for an aggressiveness between $x / c=0.30$ and $x / c=0.37$. The cavitating flow is the most aggressive when the cavity is detached by the re-entrant jet (from $t_{f}^{*}=0.47$ to $t^{*} f=1.01$ in Fig. 11) and the cavitating cloud is collapsing.

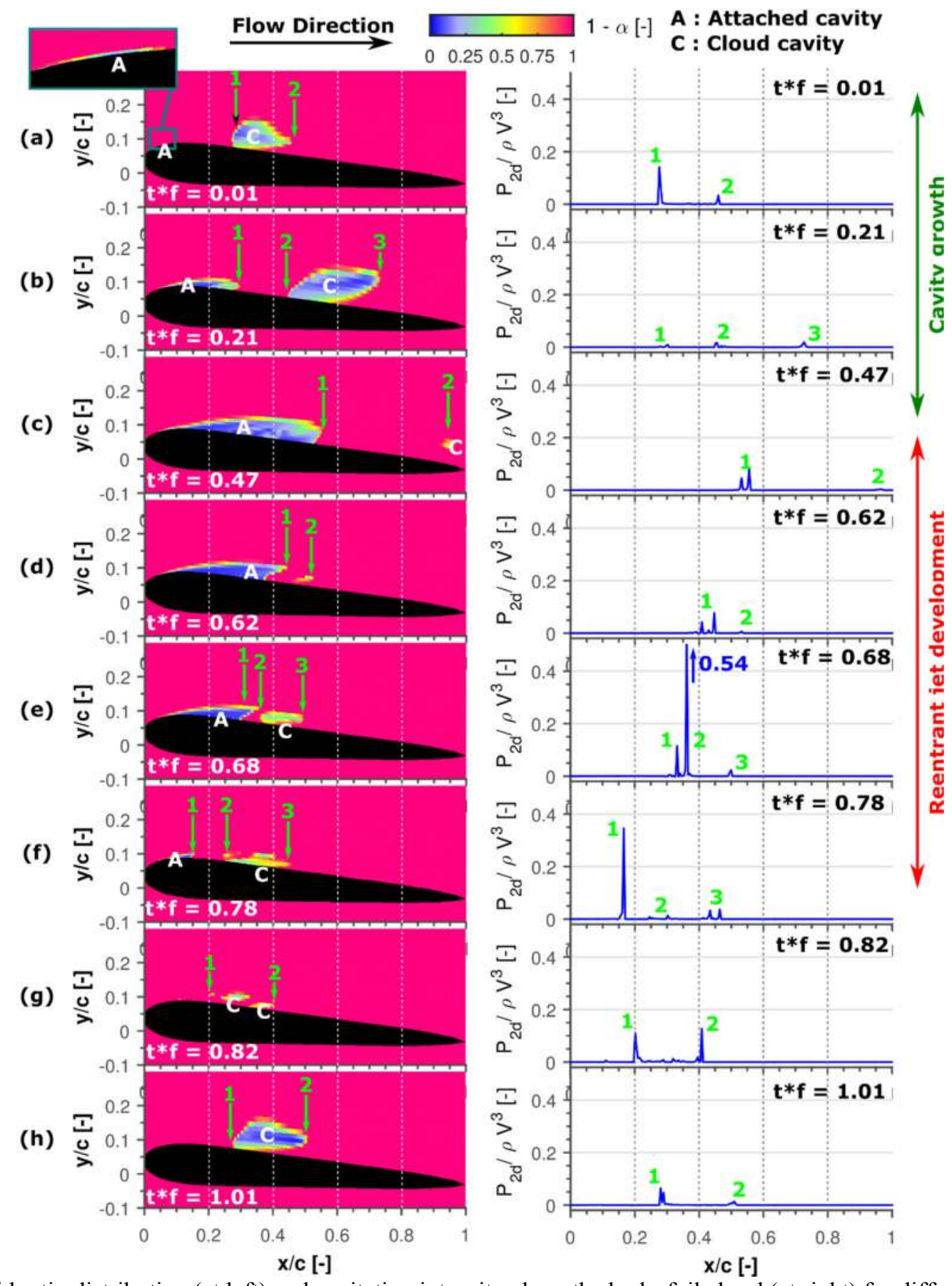

Fig. 11 Void ratio distribution (at left) and cavitation intensity along the hydrofoil chord (at right) for different time steps during a shedding cycle

( $\sigma=1.1, V=8 \mathrm{~m} / \mathrm{s}$ and angle of attack: 4 deg.).

According to Fig. 10, the instantaneous aggressiveness is periodic and three regions of high aggressiveness can be distinguished. The region I, for $x / c \leq 0.40$ in Fig. 10, corresponds to the collapse of an attached small cavity observed at $t^{*} f=0.78$ in Fig. 11 and of the upstream part of the shed cloud at $t^{*} f=0.01$ or $t^{*} f=1.01$ in Fig. 11. In comparison with experimental observations, 2D numerical predictions generally overestimate the flow aggressiveness in this region [25]. 
(a)

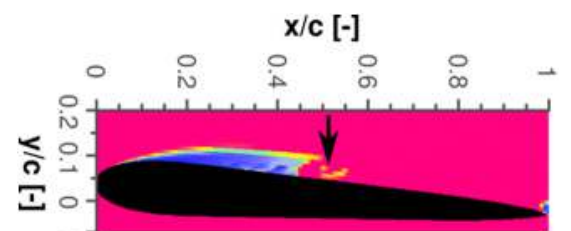

(b)
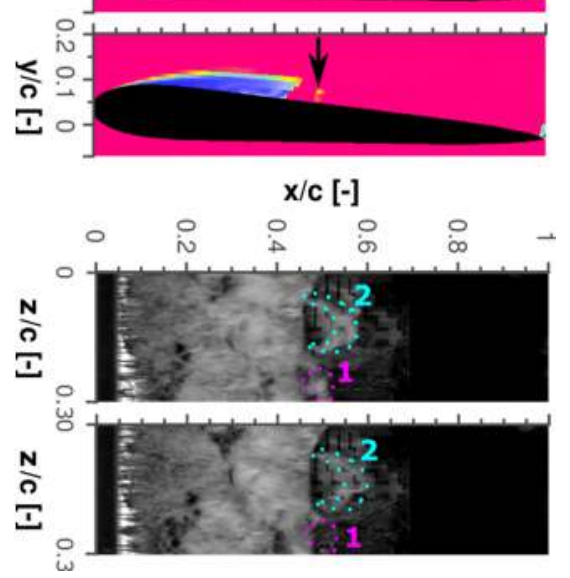

Fig. 12 Structure collapsing at the cavity closure, (a) numerical void ratio distributions $V=8 \mathrm{~m} / \mathrm{s}$ and (b) experimental pictures $V=10 \mathrm{~m} / \mathrm{s}$.

( $\sigma=1.1$ and angle of attack: 4 deg., $l / c \sim 0.55 \pm 0.05$ ). (a)
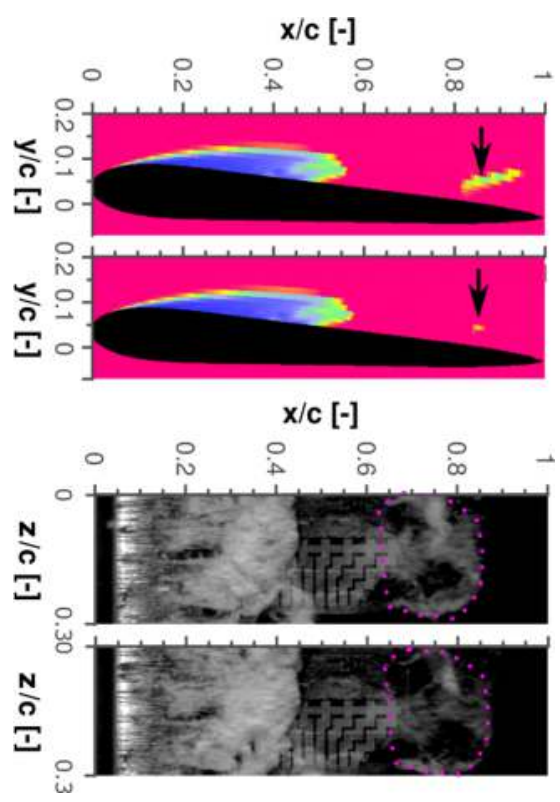

Fig. 13 Collapsus of shed cloud, (a) numerical void ratio distributions $V=8 \mathrm{~m} / \mathrm{s}$ and (b) experimental pictures $V=10 \mathrm{~m} / \mathrm{s}$.

( $\sigma=1.1$ and angle of attack: 4 deg., $/ / c \sim 0.55 \pm 0.05$ ).

The region II (Fig. 10) is located around the hydrofoil mid-chord, in the cavity closure region, where the aggressiveness intensity and the damage risks are maximum. This aggressiveness is probably due to the re-entrant jet development and the creation of cavitating structures in the cavity closure region which collapse, such as the one indicated by a black arrow in Fig. 12(a). Similar behavior is observed experimentally in Fig. 12(b), with a small bubble cloud (number 1 in Fig. 12(b)) and a horseshoe structure (number 2 in Fig. 12(b)) collapsing around the cavity closure.

In region III (Fig. 10), near the trailing edge, the flow aggressiveness is due to the collapse of the shed clouds observed between times $t^{*}=0.21$ and $t^{*} f=0.47$ in Fig. 11 or in Fig. 13(a). Experimentally in Fig. 13(b) a shed cloud surrounded by a pink dotted line is collapsing near the trailing edge.

\section{Comparison between experimental and numerical results}

Figure 13 presents a comparison between experimental measurements and numerical predictions of the cavitation aggressiveness for a constant cavitation number (close to 1), a constant angle of attack (4 deg.) and two different velocities $8 \mathrm{~m} / \mathrm{s}$ and $10.4 \mathrm{~m} / \mathrm{s}$. Right scale shows the computed non-dimensional aggressiveness whereas left scale shows the measured peak rate. Both are plotted as a function of the non-dimensional distance from the hydrofoil leading edge. Numerical and experimental sensors have same areas $(2 \mathrm{~mm} \times 2 \mathrm{~mm})$ and locations.

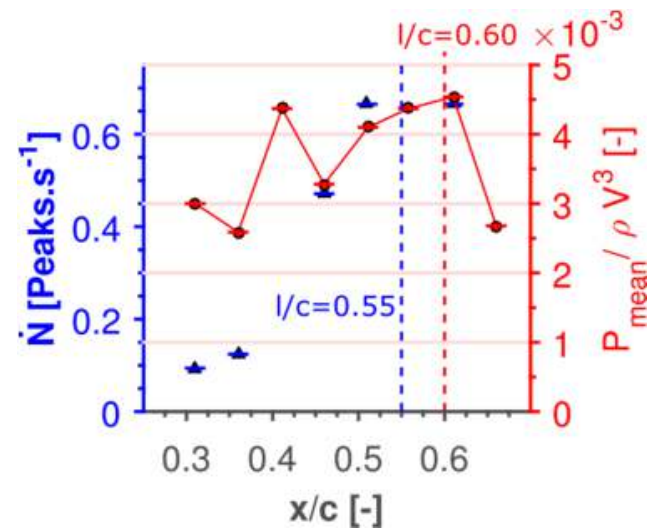

(a)

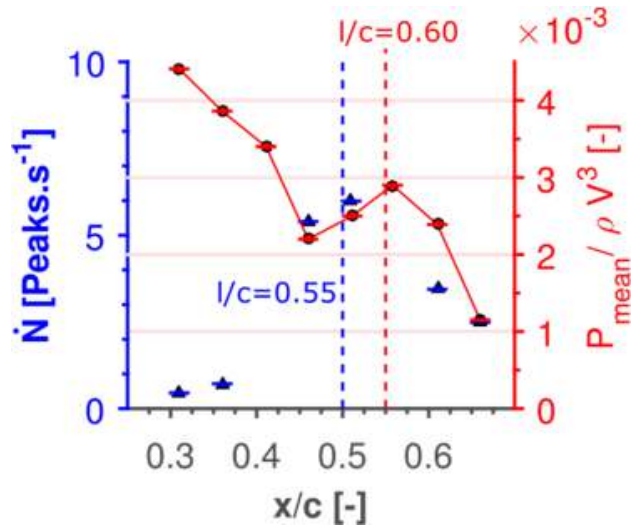

(b)

Fig. 14 Mean values of aggressiveness intensity calculated along the hydrofoil chord - qualitative comparison with the experimental peak rate. (a) $V=8 \mathrm{~m} / \mathrm{s}$, (b) $V=10.4 \mathrm{~m} / \mathrm{s}$. $(\sigma \approx 1$, angle of attack: 4 deg. $)$

Numerically, the mean aggressiveness is overestimated for $x / c \leq 0.40$ (region I in Fig. 10), as observed previously from 2D calculations [25]. The prediction of the aggressive region for $x / c \geq 0.40$ (region II in Fig. 10) appears to be in good agreement with the experimental measurements. For both cases and for both experimental and numerical results, the most critical zone corresponds to the closure region of the leading edge cavity (region II in Fig. 10). Moreover, the predicted width of the critical zone is very 
similar between experimental and numerical results. At this step, however, the experimental and numerical values of the aggressiveness cannot be compared quantitatively since they do not represent values of the same physical quantity.

\section{Conclusion}

A hydrofoil instrumented with PVDF pressure sensors was tested in the LEGI hydrodynamic tunnel under different cavitating conditions in order to estimate cavitation aggressiveness. These pressure sensors were able to resist to cavitation and to measure the pressure peaks due to the collapse of cavitating structures.

The measurements were compared to flow aggressiveness evaluated by a 2D numerical approach. Despite 3D effects clearly visible in the experiments (see for example Fig. 3(a)), the numerical simulations led to a good prediction of the cloud shedding process (including shedding frequency) and the extension of the aggressive area at cavity closure. Conversely, CFD unsteady results near the leading edge $(\mathrm{x} / \mathrm{c}<0.40)$ disagreed with experimental observations since the aggressiveness predicted in this area was overestimated. It should be observed that Leclercq et al. [26], using the same aggressiveness model but based on 3D computations, did not observe this overestimation. In any case, the proposed aggres siveness model, associated with suitable CFD tools, represents a promising approach to predict the erosive potential of a cavitating flow in real hydraulic systems and machinery.

Experimentally, the next step would be to instrument the foil with pressure sensors along the complete chord in order to capture the collapse of the shed clouds and evaluate the contribution of cloud cavitation to the erosive potential. Finally, simultaneous recording of the pressure peaks and high speed videos could provide further information on the cavitating structures responsible for aggressiveness.

\section{Acknowledgments}

The authors gratefully acknowledge the Foundation Grenoble INP through the industrial chair GE (General Electric) "HYDRO'LIKE". The code "IZ" was developed at LEGI with the support of CNES (Centre National d'Etudes Spatiales). The authors thank as well the laboratory technical staff and particularly Mile Kusulja, Muriel Lagauzère, Michel Riondet and Tristan Vandenberghe for their help for designing and building the test section. The authors are very grateful to Timo Bouvard who developed tools for post-treating the CFD results during an internship at LEGI.

\section{Nomenclature}

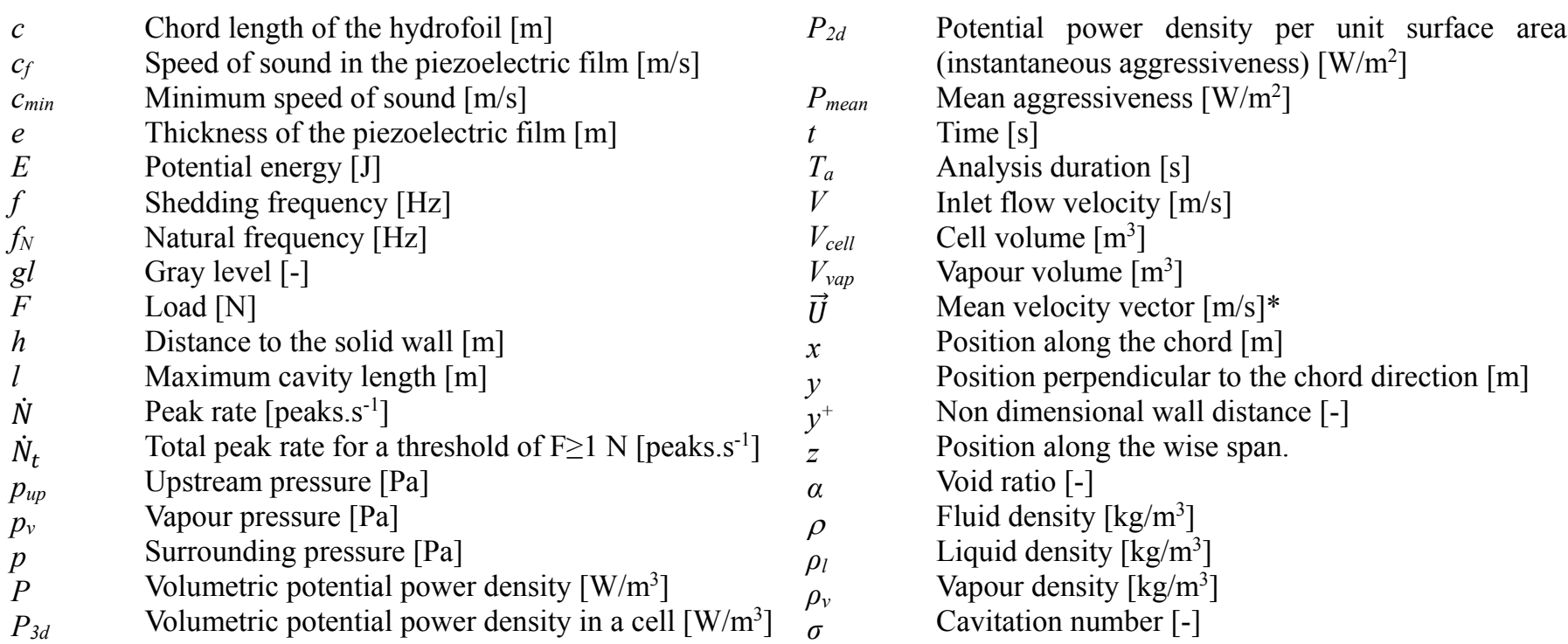

\section{References}

[1] Knapp, R. T., 1958, “Accelerated Field Tests of Cavitation Intensity,” Trans. ASME, p. 12.

[2] Fortes-Patella, R., Reboud, J. L., and Archer, A., 2000, "Cavitation Damage Measurement by 3D Laser Profilometry,” Wear, 246(1), pp. 59-67.

[3] Belahadji, B., Franc, J.-P., and Michel, J.-M., 1991, “A Statistical Analysis of Cavitation Erosion Pits,” J. Fluids Eng., 113(4), pp. 700-706.

[4] Pöhl, F., Mottyll, S., Skoda, R., and Huth, S., 2015, "Evaluation of Cavitation-Induced Pressure Loads Applied to Material Surfaces by Finite-Element-Assisted Pit Analysis and Numerical Investigation of the Elasto-Plastic Deformation of Metallic Materials," 20th Int. Conf. Wear Mater., 330-331, pp. 618-628.

[5] Roy, S. C., Franc, J.-P., and Fivel, M., 2015, "Cavitation Erosion: Using the Target Material as a Pressure Sensor,” J. Appl. Phys., 118(16), p. 164905.

[6] Kim, K. H., Chahine, G., Franc, J. P., and Karimi, A., eds., 2014, Advanced Experimental and Numerical Techniques for 
Cavitation Erosion Prediction, Springer Netherlands, Dordrecht.

[7] Hammitt, F. G., 1979, “Cavitation Erosion: The State of the Art and Predicting Capability.,” Appl. Mech. Rev., 32(6), pp. 665675.

[8] De, M. K., and Hammitt, F. G., 1982, "New Method for Monitoring and Correlating Cavitation Noise to Erosion Capability," J. Fluids Eng., 104(4), pp. 434-441.

[9] Mihatsch, M. S., Schmidt, S. J., and Adams, N. A., 2015, "Cavitation Erosion Prediction Based on Analysis of Flow Dynamics and Impact Load Spectra," Phys. Fluids, 27(10), p. 103302.

[10] Hattori, S., Mori, H., and Okada, T., 1998, “Quantitative Evaluation of Cavitation Erosion,” J. Fluids Eng., 120(1), pp. 179185.

[11] Kang, C., Liu, H., and Soyama, H., 2018, "Estimation of Aggressive Intensity of a Cavitating Jet with Multiple Experimental Methods,"Wear, 394-395, pp. 176-186.

[12] Carrat, J.-B., Fortes-Patella, R., and Franc, J.-P., 2017, “Assessment of Cavitating Flow Aggressiveness on a Hydrofoil: Experimental and Numerical Approaches," ASME, p. V01AT05A013.

[13] Fortes-Patella, R., Archer, A., and Flageul, C., 2012, "Numerical and Experimental Investigations on Cavitation Erosion," IOP Conf. Ser. Earth Environ. Sci., 15(2), p. 022013.

[14] Brown, L. F., 2000, "Design Considerations for Piezoelectric Polymer Ultrasound Transducers," IEEE Trans. Ultrason. Ferroelectr. Freq. Control, 47(6), pp. 1377-1396.

[15] Soyama, H., Lichtarowicz, A., Momma, T., and Williams, E. J., 1998, “A New Calibration Method for Dynamically Loaded Transducers and Its Application to Cavitation Impact Measurement," J. Fluids Eng., 120(4), p. 712.

[16] Dular, M., and Petkovšek, M., 2015, "On the Mechanisms of Cavitation Erosion - Coupling High Speed Videos to Damage Patterns," Exp. Therm. Fluid Sci., 68, pp. 359-370.

[17] Fujisawa, N., Kikuchi, T., Fujisawa, K., and Yamagata, T., 2017, “Time-Resolved Observations of Pit Formation and Cloud Behavior in Cavitating Jet," Wear, 386-387, pp. 99-105.

[18] Dimitrov, M., Franz Pelz, P., Lyashenko, A., Hakimi Tehrani, A., and Dörsam, E., 2014, "Measurement System by Printed Thin Pressure Sensor Array," 9th International Fluid Power Conference, Aachen.

[19] Franc, J. P., 2009, "Incubation Time and Cavitation Erosion Rate of Work-Hardening Materials,” J. Fluids Eng., 131(2), p. 021303.

[20] Gavaises, M., Villa, F., Koukouvinis, P., Marengo, M., and Franc, J. P., 2015, "Visualisation and LES Simulation of Cavitation Cloud Formation and Collapse in an Axisymmetric Geometry," Int. J. Multiph. Flow, 68, pp. 14-26.

[21] Le, Q., Franc, J. P., and Michel, J. M., 1993, "Partial Cavities: Pressure Pulse Distribution around Cavity Closure,” J. Fluids Eng., 115(2), pp. 249-254.

[22] Zima, P., Müller, M., Hujer, J., Václav, J., Sedlář, M., and Komárek, M., 2016, “Measurement of Impact Loads in a Cavitating Flow on a NACA 2412 Hydrofoil," 4th Cavitation Workshop, Chania, Grèce.

[23] Coutier-Delgosha, O., Reboud, J. L., and Delannoy, Y., 2003, "Numerical Simulation of the Unsteady Behaviour of Cavitating Flows," Int. J. Numer. Methods Fluids, 42(5), pp. 527-548.

[24] Coutier-Delgosha, O., Fortes-Patella, R., and Reboud, J. L., 2003, "Evaluation of the Turbulence Model Influence on the Numerical Simulations of Unsteady Cavitation," J. Fluids Eng., 125(1), pp. 38-45.

[25] Flageul, C., Fortes-Patella, R., and Archer, A., 2012, "Cavitation Erosion Predicted by Numerical Simulations," 14th International Symposium on Transport Phenomena and Dynamics of Rotating Machinery, ISROMAC-14, Honolulu, HI, USA.

[26] Leclercq, C., Archer, A., and Fortes-Patella, R., 2016, "Numerical Investigations on Cavitation Intensity for 3D Homogeneous Unsteady Viscous Flows," IOP Conf. Ser. Earth Environ. Sci., 49(9), p. 092007.

[27] Vogel, A., Lauterborn, W., and Timm, R., 1989, "Optical and Acoustic Investigations of the Dynamics of Laser-Produced Cavitation Bubbles near a Solid Boundary,” J. Fluid Mech., 206(1), p. 299.

[28] Petkovšek, M., and Dular, M., 2013, "Simultaneous Observation of Cavitation Structures and Cavitation Erosion," Wear, 300(1-2), pp. 55-64. 